\title{
Electric field effect on the diffusion modified AIGaAs/GaAs single quantum well
}

\author{
Sudhira Panda, ${ }^{\text {a) }}$ B. K. Panda, S. Fung, and C. D. Beling \\ Department of Physics, The University of Hong Kong, Hong Kong
}

(Received 18 September 1995; accepted for publication 15 April 1996)

\begin{abstract}
The electron subband energies and wave functions in an interdiffusion-induced $\mathrm{Al}_{x} \mathrm{Ga}_{1-x} \mathrm{As} /$ GaAs/ $/ \mathrm{Al}_{x} \mathrm{Ga}_{1-x} \mathrm{As}$ single quantum well are calculated in the presence of the dc electric field using the finite difference method. The mean lifetimes are obtained from the time-dependent probability of tunneling of the wave packet out of the well by the applied electric field. The effect of the applied electric field on the subband energies in the well is the same as in the as-grown square quantum well when the interdiffusion length is below $20 \AA$. In the well with higher diffusion length the barrier height reduces so that the wave function tunnels out of the well. The linear and nonlinear intersubband absorption coefficients and the change in the real part of the index of refraction are calculated with the applied electric field at $100 \mathrm{kV} / \mathrm{cm}$ and without the field in both the as-grown square well and the diffusion modified well with the interdiffusion length at $20 \AA$ A. (C) 1996 American Institute of Physics. [S0021-8979(96)06414-6]
\end{abstract}

\section{INTRODUCTION}

Quantum wells (QWs) have promised a lot of device applications such as modulation-doped field-effect transistors and quantum well lasers. ${ }^{1,2}$ In addition to this, QWs have been found to play a key role in optoelectronics applications such as infrared detectors, wave guides, fast switch and others. ${ }^{3}$ The main difference between the QW and the bulk semiconductor is that the electrons are confined in a twodimensional space in the QW and along the grown direction several discreet energy levels are formed. The position of the Fermi level in the conduction band is decided by the nature of doping and the temperature of the sample. For optoelectronics application studies it is needed to study the absorption of the incident photon by carriers under the applied electric field for which it is required to obtain the subband energies and wave functions under the applied electric field. ${ }^{4}$ The knowledge of the mean tunneling carrier lifetime and other transport properties such as the phonon scattering and interface roughness scattering are quite essential for understanding the operating priciples of QW devices. ${ }^{5}$

The QWs grown in the molecular beam expitaxy method have square well shape. When the as-grown square quantum well (SQW) is subjected to annealing above $800^{\circ} \mathrm{C}$ the intermixing at the heterojunction starts. ${ }^{6}$ Chang and Koma have found that the activation energy required for the interdiffusion process is $4.1 \mathrm{eV}^{6}$ In their experiment they did not take care of the As vacancy formation in the sample at high annealing temperature. Recently Mukai, Sugawara and Yamazaki $^{7}$ carefully carried out the annealing with a cap layer on the sample to minimise As vacancy formation and found that the activation energy required for the interdiffusion process is $2.8 \mathrm{eV}$ which is much less than that found previously by Chang and Koma. ${ }^{6}$ This is because the polarity of the As vacancies do not allow $\mathrm{Al}$ atoms to diffuse. Recently Oh et al. have measured activation energy for the Ga vacancy formation to be $2.72 \mathrm{eV}$ in the similar sample with a

${ }^{a)}$ Electronic mail: panda@hkucc.hku.hk cap layer. ${ }^{8}$ The activation energy of the interdiffusion process is thus quite similar to the activation energy of the $\mathrm{Ga}$ vacancy formation. This suggests that the formation of the $\mathrm{Ga}$ vacancy induces $\mathrm{Al}$ atoms to diffuse into the GaAs layer. Therefore the interdiffusion process is characterised by the Al diffusion length $\left(L_{d}\right)$ which can be obtained from the diffusion constant at the annealing temperature and the annealing time $(\sqrt{D t})$. Assuming the interdiffusion coefficient to be the same in GaAs and AlGaAs layers in the GaAs/ $\mathrm{Al}_{0.25} \mathrm{Ga}_{0.75} \mathrm{As}$ single quantum well the diffusion constant is found to be $4.0 \times 10^{-18} \mathrm{~cm}^{2} / \mathrm{s}$ at $900^{0} \mathrm{C}$ by Mukai, Sugawara and Yamazaki. ${ }^{7}$ With this diffusion constant the annealing times required to obtain diffusion lengths $10,20,30$ and $40 \AA$ are $0.7,2.8,6.25$ and 11.1 hours respectively. At $850{ }^{\circ} \mathrm{C}$ the diffusion constant is $2.3 \times 10^{-18} \mathrm{~cm}^{2} / \mathrm{s}$ so that much higher annealing times are required to get the diffusion lengths. With known $\mathrm{Al}$ diffusion length the confinement profile in a diffusion modified quantum well (DMQW) can be modelled. ${ }^{6,9,10}$

The energies, wave functions and mean tunneling lifetimes in the SQW in the presence of the applied electric field have been studied using Airy functions in an analytic method. ${ }^{11}$ The numerical methods include the finite difference method, ${ }^{12}$ finite element method ${ }^{13}$ and transfer matrix method. ${ }^{14}$ The diffusion modified quantum well (DMQW) for its complicated potential structure cannot be solved by an analytic method. The numerical technique such as the transfer-matrix method is quite popular for its computational simplicity to give both energies and wave functions. ${ }^{15}$ But this method involves the multiplication of a large number of matrices which unless carefully designed accumulates numerical errors giving unstable results. However, the finite difference scheme is simple and is quite efficient to yield very accurate results for any form of the potential. ${ }^{2}$ Therefore we have adopted this scheme for the present calculation.

Generally the mean-lifetime is calculated from the timeindependent effective mass equation using the transfermatrix method. ${ }^{15}$ The results of the transfer matrix method 
are not reliable for the reason already mentioned. We have adopted the method of Juang et al. ${ }^{12}$ and Panda and $\mathrm{Li}^{16}$ which solves the time-dependent Schrödinger equation in the presence of the applied electric field and then calculates the mean lifetime from the time-dependent tunneling probability.

The linear and non-linear intersubband optical absoption spectra depend on energies and wave functions and mean tunneling lifetime. ${ }^{17}$ In a SQW there are extensive experimental and theoretical studies with different sample conditions such as temperature, doping and illumination. ${ }^{18} \mathrm{Com}-$ pared to the SQW the experimental and theoretical studies are limited in diffusion induced QW. The theoretical nonlinear intersubband optical absorption at different $L_{d}$ without applied electric field by Li et al. ${ }^{19}$ have shown that it is quite strong compared to the as-grown SQW. The aim of the present work is to study the effect of the electric field on both optical absorption and the change in the dielectric constant using calculated energies and wave functions.

The methods of solving the time-independent and timedependent effective mass equation in the presence of an electric field is described in Sec. II. The expressions for the nonlinear optical absorption and the change in the refractive index are also given in Sec. II. In Sec. III the quantum confined Stark effect for different well widths at different field strengths are calculated and the mean lifetimes for the corresponding well widths and fields are shown and the main relevant features are discussed. The results of the absorption coefficient and the change in the real part of the refractive index in the DMQW are compared with those in SQW. In Sec. IV we conclude the present work.

\section{COMPUTATIONAL METHODS}

For simplicity the interdiffusion of $\mathrm{Al}$ and $\mathrm{Ga}$ atoms is assumed to be isotropic at the same temperature. There are two different expressions available to get the $\mathrm{Al}$ concentration profile in terms of the characteristic $\mathrm{Al}$ diffusion length $L_{d}$. The concentration profile found from the diffusion equation is error functional in nature. This form has been adopted by a number of researchers ${ }^{6,9,10,20}$ to calculate subband energies and wave functions in the DMQW. Lahiri et al. have expressed the $\mathrm{Al}$ concentration profile in a polynomial with three parameters such as well width, barrier thickness, and Al diffusion length. ${ }^{21}$ They have not defined another parameter $h$ in their expression for the $\mathrm{Al}$ concentration profile. They have also not mentioned how they have obtained such an expression. Therefore we have taken the error functional form of the $\mathrm{Al}$ concentration profile which is well understood from the diffusion equation and is given by

$$
w(z)=x\left\{1-\frac{1}{2}\left[\operatorname{erf}\left(\frac{L+2 z}{4 L_{d}}\right)+\operatorname{erf}\left(\frac{L-2 z}{4 L_{d}}\right)\right]\right\}
$$

where $L$ is the as-grown width of the SQW and erf is the error function. ${ }^{22}$ The confinement potential created by the interdiffusion process is expressed as

$$
V(z)=B_{\text {off }}\left\{E_{g}[w(z)]-E_{g}[w(0)]\right\},
$$

where $B_{\text {off }}$ is the band offset splitting ratio taken to be 0.7 and $E_{g}(w)$ is the bandgap which for the annealed material at position dependent $\mathrm{Al}$ composition is taken as ${ }^{20}$

$$
E_{g}(w)=1.424+1.594 w+w(1-w)(0.127-1.31 w) e V .
$$

The subband energies and wave functions can be calculated using single band effective mass equation given by BenDaniel and Duke. ${ }^{23}$ Interdiffusion causes lattice strain in the barrier region. ${ }^{7}$ We have not taken this correction in the present calculation.

$$
\left[-\frac{\hbar^{2}}{2} \frac{\partial}{\partial z}\left(\frac{1}{m^{*}(z)} \frac{\partial}{\partial z}\right)+V(z)+|q| F z\right] \Psi(z)=E \Psi(z) .
$$

The band non-parabolicity is usually taken into account in the energy dependent effective mass. We have not considered the band non-parabolicity in the present calculation. The position dependent room temperature effective mass is taken $\mathrm{as}^{20}$

$$
m^{*}(z)=[0.0665+0.0835 w(z)] m_{0},
$$

where $m_{0}$ is the electron rest mass. We have employed the finite difference method to solve this equation to get both energies and wave functions. ${ }^{2,16}$ In this method the total width $d$ which is the combination of the well width and the width of the barrier is divided into $N$ parts with each part ( $\epsilon)$ equal to $d /(N-1)$. With the center of the QW taken at $z=0$ the wave function can be described at each $z$ point in the column matrix form ${ }^{2} \Psi_{j}=\Psi[-(d / 2)+(j-1) \epsilon]$. The differential operator in Eq. (2.4) can be written in the finite difference matrix to obtain a matrix in the form

$$
\bar{H} \Psi=E \Psi,
$$

where $\bar{H}$ is a $N \times N$ is a real symmetric tridiagonal matrix and $\Psi$ is a column matrix. Usually the matrix Eq. (2.6) is diagonalised to obtain both energies and wave function. ${ }^{2}$ While setting the finite difference scheme the boundary condition is that $\Psi( \pm d / 2)=0$. It will be shown later that the diagonalisation scheme gives incorrect eigenvalues and eigenfunctions when the well is under large electric field. In order to get the energies and wave functions at both low and high applied field regions we have adopted the inverse power method $^{24}$ which has been found to be successful employed in the SQW. ${ }^{12,16}$ In this method the following self-consistent equations are solved iteratively to obtain energies and wave functions.

$$
\begin{aligned}
& \Psi^{(k)}=\left[\bar{H}-E^{(k-1)} I\right]^{-1} \Psi^{(k-1)}, \\
& E^{(k)}=\left\langle\Psi^{(k)}|\bar{H}| \Psi^{(k)}\right\rangle,
\end{aligned}
$$

where $I$ is the identity matrix. This iterative method needs an input guess energy and wave function as the starting point. We first diagonalise Eq. (2.6) without the electric field to get the energy and wave function. Then in Eqs. (2.7) and (2.8) the electric field effect is put in $\bar{H}$ and the zero field energy and wave function are used as starting values to solve Eqs. (2.7) and (2.8). This method as it will be shown later is capable of obtaining the oscillations of the tunnelled wave 
function in the barrier region due to high electric field which is not possible in the diagonalisation scheme.

The formalism for the tunneling of the wave packet out of the ultrathin well by the applied electric field has been first studied by Kundrotas and Dargys. ${ }^{25}$ Juang et al. have extended this method to the wide quantum well. ${ }^{12}$ In this method the well without any applied bias is assumed to have bound states and a continuum of free states characterised by the wave vector $\mathbf{k}$. The tunneling process is initiated by suddenly applying electric field at $t=0$. The time-dependent wave function at a particular bound state energy is obtained from the time-dependent Schrödinger equation as

$$
\begin{gathered}
{\left[-\frac{\hbar^{2}}{2} \frac{\partial}{\partial z}\left(\frac{1}{m^{*}(z)} \frac{\partial}{\partial z}\right)+V(z)+|q| F z\right] \Psi_{E}(z, t)} \\
=i \hbar \frac{d}{d t} \Psi_{E}(z, t) .
\end{gathered}
$$

We have adopted the finite difference method of Juang et al. ${ }^{12}$ to solve this equation. In this method $\Psi_{E}(z, t)$ is discretised as $\Psi_{i, n}=\Psi[-(d / 2)+(i-1) \epsilon, n \delta]$ with $\delta$ and $n$ as the time spacing and time index respectively. In this method Eq. (2.9) can be expressed in a standard $A c=b$ matrix form with $A$ as a complex triangular square matrix, and $c$ and $b$ are both complex column matrices. It is solved by the Gaussian elimination and back substitution method. Since the bias is turned on at $t=0$ the initial wave function $\Psi_{E}(z, 0)$ is taken to be the time-independent wave function with zero bias for the eigenvalue $E$.

The probability of tunneling which is defined as the number of electrons tunneled out at time $t$ can be calculated by projecting $\Psi_{E}(z, t)$ on all possible free electron wave functions $\Psi_{k}(z, t)$,

$$
\begin{aligned}
P_{E}(t) & =\frac{1}{2 \pi} \int_{-\infty}^{\infty} d k\left|\left\langle\Psi_{\mathbf{k}}(z, t) \mid \Psi_{E}(z, t)\right\rangle\right|^{2} \\
& =1-\left|\left\langle\Psi_{E}(z, 0) \mid \Psi_{E}(z, t)\right\rangle\right|^{2} .
\end{aligned}
$$

From this expression we can observe that at $t=0$, the $P_{E}(t)=0$ because the bias is switched on at this time. At large $t,\left|\left\langle\Psi_{E}(z, 0) \mid \Psi_{E}(z, t)\right\rangle\right|^{2}$ can become zero showing that the tunneling process is complete.

When the optical intensity is put on the QW which is under the applied electric field, there will be linear and nonlinear polarisations. In addition to this there will be a dc rectification term and second and higher harmonic generation terms. ${ }^{26}$ Neglecting the dc rectification and the harmonic generation terms the polarisation is expressed in terms of the linear and non-linear susceptibilities as

$$
\begin{aligned}
P(t)= & \epsilon_{0} \chi^{(1)}(\omega) I_{\mathrm{op}} e^{-i w t}+\epsilon_{0} \chi^{(3)}(\omega) I_{\mathrm{op}} e^{-i w t}+\epsilon_{0} \chi^{(1)} \\
& \times(-\omega) I_{\mathrm{op}}^{*} e^{i w t}+\epsilon_{0} \chi^{(3)}(-\omega) I_{\mathrm{op}}^{*} e^{i w t}
\end{aligned}
$$

where $I_{\mathrm{op}}$ is the amplitude of the optical source. The analytic term for the linear susceptibility $\chi^{(1)}(\omega)$ as derived by Ahn and Chuang ${ }^{17}$ and Kuhn et al. ${ }^{18}$ is given by

$$
\begin{aligned}
\epsilon_{0} \chi^{(1)}(\omega)= & \left|M_{f i}\right|^{2}\left(\frac{m^{*} k_{B} T}{L_{\mathrm{eff}} \pi \hbar^{2}}\right) \\
& \times \ln \left(\frac{1+\exp \left[\left(E_{F}-E_{i} / k_{B} T\right]\right.}{1+\exp \left[E_{F}-E_{f} / k_{B} T\right]}\right) \\
& \times \frac{1}{E_{f}-E_{i}-\hbar w-i\left(\hbar / \tau_{i f}\right)}
\end{aligned}
$$

where $E_{i}, E_{f}$ and $E_{F}$ are respectively the initial, final and Fermi energies. $m^{*}$ is the effective mass in the quantum well. $L_{\text {eff }}$ is the effective width of the well. In a SQW the $L_{\text {eff }}$ is just $L$ whereas in the diffusion-modified QW the $L_{\text {eff }}$ is different from $L$. We will give the definition of $L_{\text {eff }}$ in a diffusion-modified QW later in the next section. $M_{a b}$ is the dipole matrix element between states $|a\rangle$ and $|b\rangle$ described as

$$
M_{a b}=|q| \int \Psi_{a}(z) z \Psi_{b}(z) d z .
$$

For arbitrary polarisation, an additional factor of $\cos \theta$ must be incorporated into the matrix element, where $\theta$ is the angle between the $z$ axis and the direction of polarisation for intersubband transitions. The dephasing rate $\tau_{a b}$ is given as

$$
\frac{1}{\tau_{a b}}=\frac{1}{\tau_{a}}+\frac{1}{\tau_{b}} .
$$

The dephasing rate is the combination of the rates from the electron tunneling time, electron-phonon scattering, ionised impurity scattering and interface roughness scattering. We have not evaluated the mean lifetimes for all these processes theoretically as done by Walukiewicz, ${ }^{28}$ but used the experimental value of $0.14 \mathrm{ps}$ which was obtained experimentally for a SQW with the barrier height $340 \mathrm{meV}$ at $77 \mathrm{~K}$. In the present work the carrier scattering with the interface roughness becomes significant because of interdiffusion process. In spite of this we have used the same value as in the SQW because we have no experimental data to prove the correctness of our calculated value.

The third order non-linear susceptibility is given by

$$
\begin{aligned}
\epsilon_{0} \chi^{(3)}(\omega)= & -\chi^{(1)}(\omega) I\left[\frac{2\left(\tau_{i i}+\tau_{f f}\right)\left|M_{f i}\right|^{2}}{\tau_{i f}\left[\left(E_{f}-E_{i}-\hbar w\right)^{2}+\left(\hbar^{2} / \tau_{i f}^{2}\right)\right]}\right. \\
& \left.-\frac{\left(M_{f f}-M_{i i}\right)^{2}}{\left[E_{f}-E_{i}-i\left(\hbar / \tau_{i f}\right)\right]\left[E_{f}-E_{i}-\hbar w-i\left(\hbar / \tau_{i f}\right)\right]}\right]
\end{aligned}
$$

where $I=\left|I_{\mathrm{op}}\right|^{2}$ is the intensity of the optical source. The susceptibility $\chi$ is related to the absorption coeeficient $\alpha(\omega, I)$ by,

$$
\alpha(\omega)=\omega\left(\frac{\mu}{\epsilon_{R}}\right)^{1 / 2} \operatorname{Im}\left[\epsilon_{0} \chi(\omega)\right]
$$

where $\epsilon_{R}$ is the real part of the permitivity. The change in the refractive index is related to the susceptibility as

$$
\frac{\Delta n(\omega)}{n_{r}}=\operatorname{Re}\left(\frac{\chi(\omega)}{2 n_{r}^{2}}\right)
$$

where $n_{r}$ is the refractive index. 


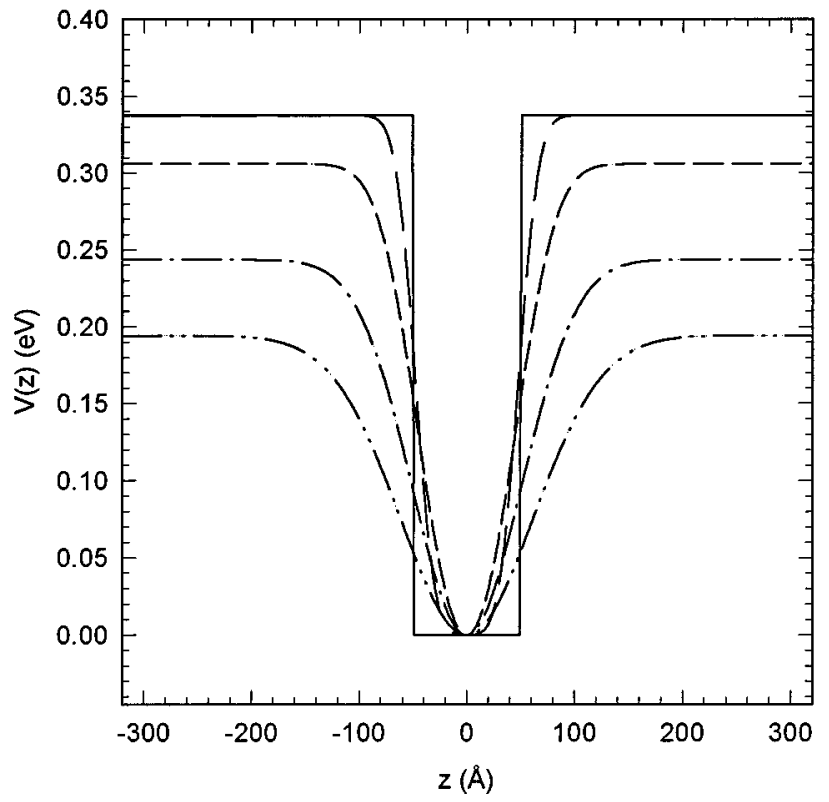

FIG. 1. Potential profiles for both the as-grown and diffusion modified single quantum well $\mathrm{Al}_{x} \mathrm{Ga}_{1-x} \mathrm{As} / \mathrm{GaAs} / \mathrm{Al}_{x} \mathrm{Ga}_{1-x} \mathrm{As}$. The $\mathrm{Al}$ concentration is taken to be $x=0.353$ so that the barrier height of the SQW is nearly 340 $\mathrm{meV}$. The width of the as-grown SQW is taken to be $100 \AA$. The solid curve represents the potential profile for the SQW corresponding to $L_{d}=0$ and the DMQWs with diffusion lengths $\left(L_{d}\right) 10 \AA, 20 \AA, 30 \AA$ and $40 \AA$ are shown by long-dashed, short-dashed, dot-dashed and dot-dot-dashed lines respectively.

\section{RESULTS AND DISCUSSIONS}

The structure of the DMQWs for different diffusion lengths $\left(L_{d}\right)$ are shown in Fig. 1. It is clear that the as-grown QW corresponding to zero diffusion length is a SQW which justifies Eq. (2.1) taken for the change of Al concentration as a function of $L_{d}$. The depth of the potential well decreases with increasing $L_{d}$. The interdiffusion process is shown as the degradation of the sharp boundary of SQW.

Our finite difference method is different from that previously employed by Li et al. to obtain the electron subband energies in the SQW and DMQW. ${ }^{20}$ Therefore it is required to compare our energies in both the SQW and DMQW with their tabulated data. We have obtained energies by taking $L=100 \AA$ and $x=0.3$ which are chosen by Li et al. For the SQW the total width $(d)$ is found to be $800 \AA$ to obtain good convergence. For this SQW we have found three eigenvalues $32 \mathrm{meV}, 126.6 \mathrm{meV}$ and $267.13 \mathrm{meV}$. Li et al. have found eigenvalues to be $40.1 \mathrm{meV}, 146.8 \mathrm{meV}$ and $274.8 \mathrm{meV}$ in the same well. Since our eigenvalues are systematically smaller than those of Li et al. for the same well, we have calculated the eigenvalues of the SQW from the roots of the dispersion equations. We have found our results in perfect agreement with the roots of the dispersion equation which confirms that our finite difference scheme is correct. Our eigenvalues for the DMQW with $L_{d}=10 \AA$ are $45 \mathrm{meV}, 157$ $\mathrm{meV}$ and $271 \mathrm{meV}$ whereas $\mathrm{Li}$ et al. have found the three eigenvalues to be $52.8 \mathrm{meV}, 167.7 \mathrm{meV}$ and $271.1 \mathrm{meV}$. This shows that the nonuniform finite difference grid of $\mathrm{Li}$ et al. does not give accurate results.

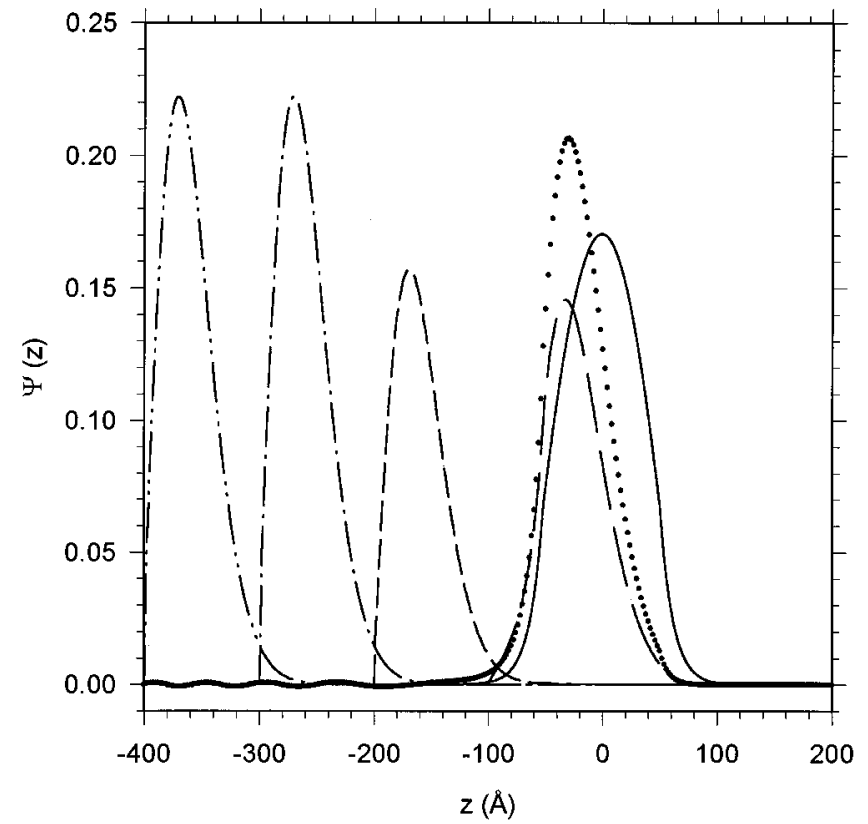

FIG. 2. Ground state wave function in an as-grown SQW as shown in Fig. 1 is obtained for different field strengths using the diagonalisation scheme in the finite difference method. The wave function at zero applied field is shown by the solid line. With the applied field strength $300 \mathrm{kV} / \mathrm{cm}$ the wave functions calculated with $d$ (the combination of the well width and barrier width) at $200 \AA, 400 \AA, 600 \AA$ and $800 \AA$ are shown by shown by long-dashed, short-dashed, dot-dashed and dot-dot-dashed lines respectively. The wave wave function calculated at $\mathrm{F}=100 \mathrm{kV} / \mathrm{cm}$ in the inverse power method with $d=800 \AA$ is shown as the dotted line. Note that the small oscillations of the wave function outside the well are due to tunneling effect. The wave function under different field strengths calculated in the inverse power scheme for DMQW is given in Fig. 7.

The wave functions obtained in the diagonalisation scheme in a SQW with $L=100 \AA$ and $x=0.353$ at the applied electric field strength $300 \mathrm{kV} / \mathrm{cm}$ for different $d$ values are shown in Fig. 2. The wave function obtained in the inverse power method is also shown in the same figure. It is observed that the position of the wave function is shifted with the increase of the $d$ value. We have taken the boundary condition in the finite difference scheme that the wave function vanishes at the boundary. This is equivalent to assuming that there are infinite barriers at $\pm d / 2$. In the absence of the external field this condition is valid because the wave function goes to zero at a finite $d$. Since the electric field is a linear function of position it creates a large triangular well at $-d / 2$. The depth of the triangular well increases with increasing $d$. When the depth of the triangular well is larger than that of the DMQW the wave function tries to localise in the triangular wave. Very recently we have obtained energies as a function of $d$ under a particular field strength using diagonalisation technique in the finite difference method. We have obtained a stabilisation graph as previously shown by Borondo and Sánchez-Dehesa employing the Airy function method $^{29}$ and by Proto et al. employing the finite difference method. ${ }^{30}$ The quasi-bound state energies and mean tunneling lifetimes can be obtained from the stabilisation graph for low applied electric fields. This work is under progress in our group and will be published elsewhere. 


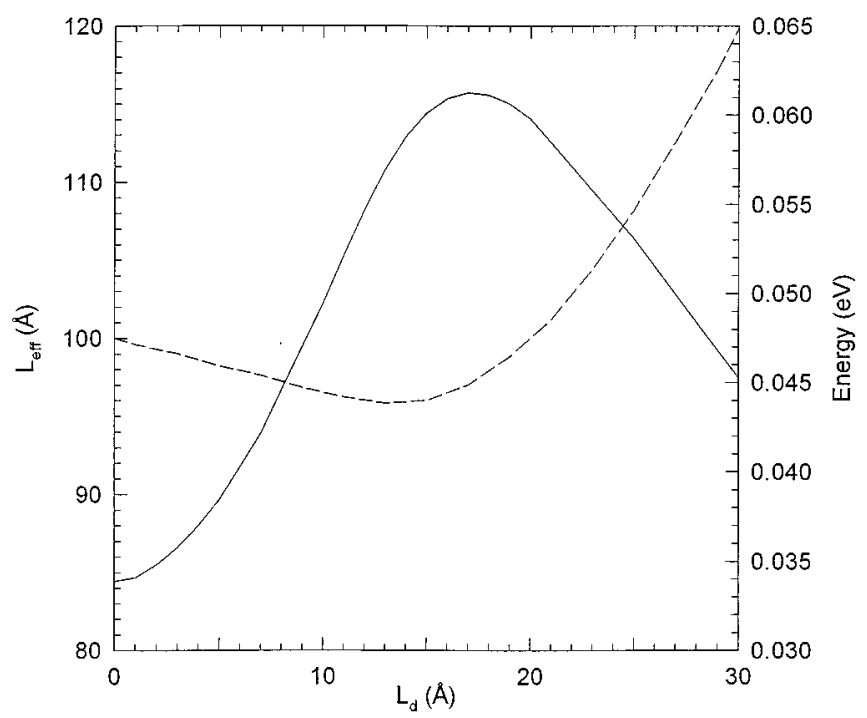

FIG. 3. Variation of the ground state energies with diffusion lengths for the quantum wells shown in Fig. 1 is represented by the solid line. The energies are shown in right axis. The effective well width $\left(L_{\text {eff }}\right)$ is calculated at the half of the barrier height for different diffusion lengths and shown as dashed line with values correspoding to the left axis.

The ground state energies for the SQW and DMQW for different $L_{d}$ at zero applied electric field are shown in Fig. 3. The energy level increases with $L_{d}$ up to $20 \AA$ and then decreases with increasing $L_{d}$. In order to understand this we have plotted the effective well width $\left(L_{\text {eff }}\right)$ which is defined as the width of the well at the half barrier height in the same figure. The $L_{\text {eff }}$ decreases slowly with increasing $L_{d}$ up to 20 $\AA$ and then increases rapidly for higher $L_{d}$. The ground state energies in the finite square well potentials are usually obtained from the roots of the energy dispersion equation. Therefore the analytic expression for the ground state energy level in a finite square well unlike in the case of an infinite square well is not known. As a result of this a direct relationship between the ground state energy level and the width of the finite square well cannot be established. But the ground state energy level in the finite square well can be matched exactly with that of the infinite square well with an increase in the width of the infinite well. ${ }^{17}$ For example, the ground state energy of an infinite quantum well with an effective well width $126.5 \AA$ agrees very well with that of a finite quantum well with width $100 \AA$ and barrier height 340 $\mathrm{meV} .{ }^{31}$ It is known that the ground state energy level in an infinite well is inversely proportional to the square of the well width. Therefore the decrease in the $L_{\text {eff }}$ without changing the barrier heights appreciably up to the diffusion length at $20 \AA$ increases the energy levels. But for DMQWs with diffusion lengths higher than $20 \AA$ the increase in the $L_{\text {eff }}$ and decrease in the barrier heights are both responsible for decreasing the ground state energies.

The effect of the electric field on the ground state energy for different $L_{d}$ are shown in Fig. 4. As it is observed in the case of the SQW previously ${ }^{16}$ the ground state energy for the DMQW with different $L_{d}$ decreases with increasing electric field. For both the as-grown and DMQW with $L_{d}$ at $10 \AA$

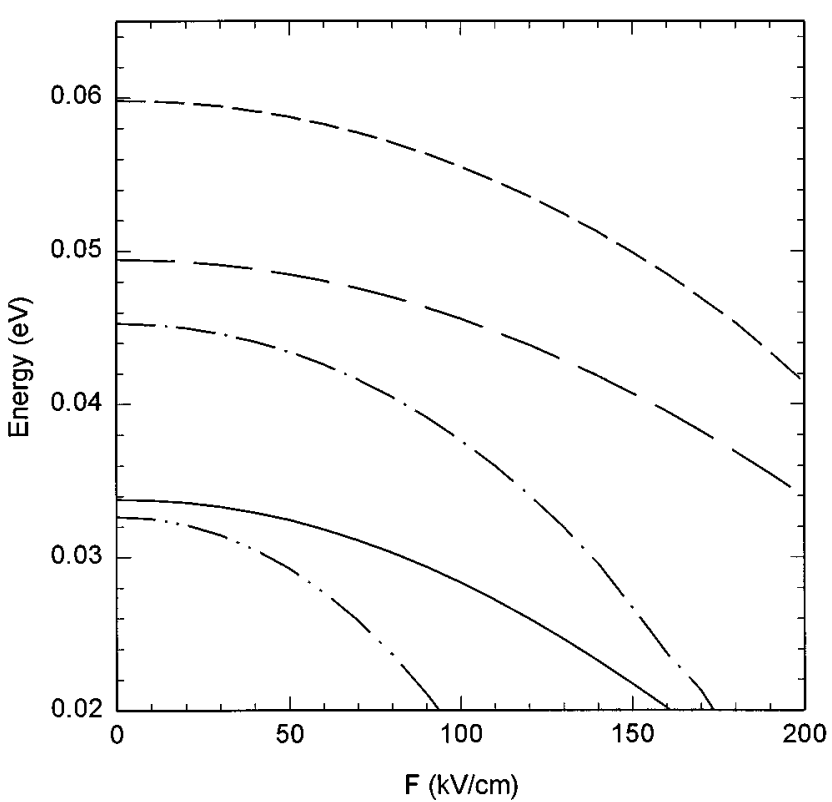

FIG. 4. Effect of the applied dc electric field on the ground state energy level of the quantum wells in Fig. 1. The wells characterised by the diffusion lengths $0 \AA, 10 \AA, 20 \AA, 30 \AA$ and $40 \AA$ are shown as soild, long-dashed, short-dashed, dot-dashed and dot-dot-dashed lines respectively.

energy levels are changed slowly within $200 \mathrm{kV} / \mathrm{cm}$, but for wells with $L_{d}$ at 20,30 and $40 \AA$ the change in the energy levels for increasing electric field is rapid. In order to understand this result the effect of the electric field on the DMQW is shown in Fig. 5. It is observed that the electric field decreases the barrier height and the bottom of the well in the direction of the electric field. At the same field strength the barrier height for the well decreases with increasing $L_{d}$. It is

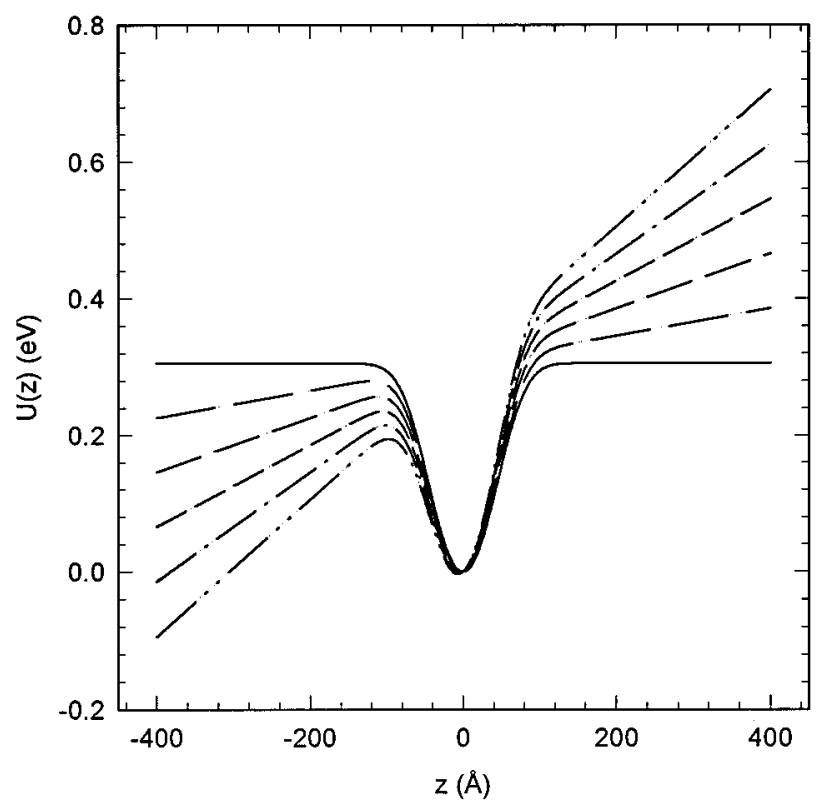

FIG. 5. Effect of the applied dc electric field on the DMQW with $L_{d}=20 \AA$. The well under field strengths $0 \mathrm{kV} / \mathrm{cm}, 20 \mathrm{kV} / \mathrm{cm}, 40 \mathrm{kV} / \mathrm{cm}, 60 \mathrm{kV} / \mathrm{cm}, 80$ $\mathrm{kV} / \mathrm{cm}$ and $100 \mathrm{kV} / \mathrm{cm}$ are denoted by solid, long-dashed, middle-dashed, short-dashed, dot-dashed and dot-dot-dashed lines respectively. 


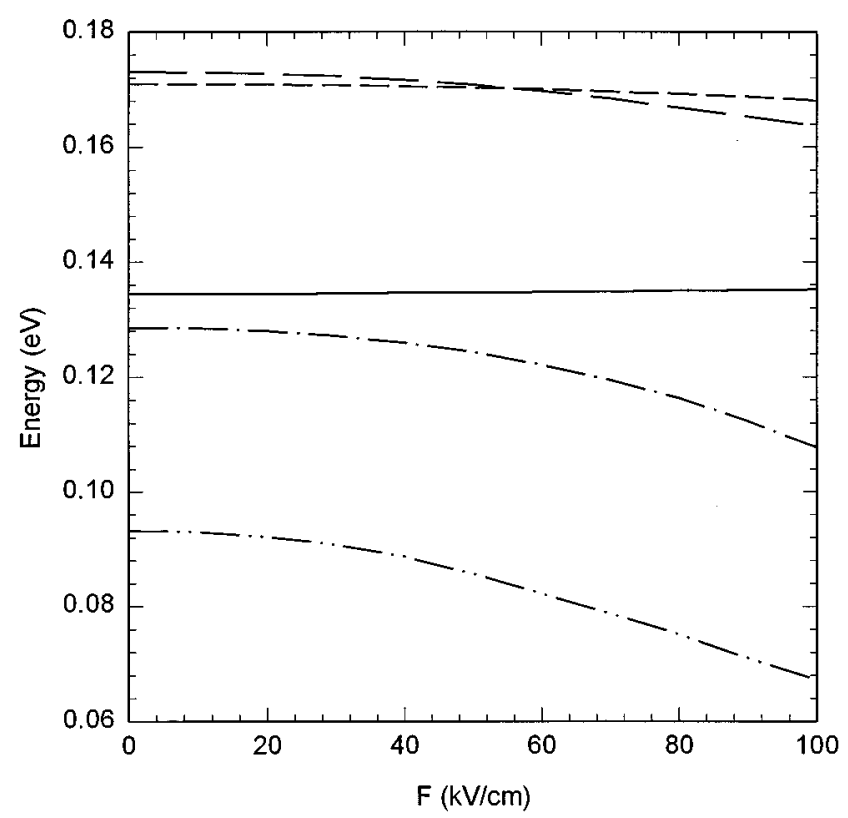

FIG. 6. Effect of the applied dc electric field on the first excited state in wells shown in Fig. 1. The energy levels for wells corresponding to $L_{d}$ at 0 $\AA$, $10 \AA, 20 \AA, 30 \AA$ and $40 \AA$ are shown as solid, short-dashed, longdashed, dot-dashed and dot-dot-dashed lines respectively.

interesting to observe that the separation between the energy levels for the SQW and DMQW with $L_{d}=10 \AA$ remain the same up to field strength $160 \mathrm{kV} / \mathrm{cm}$. The reason for such a behaviour is that the barrier heights for both the SQW and DMQW with $L_{d}=10 \AA$ are nearly the same. Since the barrier heights of the DMQWs decrease very fast with increasing electric field for higher $L_{d}$, the energy levels in these wells are also seen to decrease very fast.

The energy levels for the first excited state in both the as-grown SQW and DMQW corresponding to $L_{d}=10 \AA$, 20 $\AA$, $30 \AA$ and $40 \AA$ are plotted at different electric field strengths in Fig. 6. It is observed that without an appled field the energy level increases with increasing diffusion length up to $20 \AA$ and then decreases as observed previously in the case of the ground state energy levels. With increasing field strength the energy level increases slightly for the as-grown SQW. For the DMQW with $L_{d}=10 \AA$ the energy level decreases very slowly with increasing field strength whereas the effect of the field on the energy levels for wells with higher $L_{d}$ is quite strong. This happens because the barrier height as shown in Fig. 1 decreases with increasing $L_{d}$ and the electric field further reduces the barrier height in the direction of the electric field as shown in Fig. 5. The energy level decreases rapidly when the tunneling is quite strong. The carriers in the DMQW at $L_{d}=40 \AA$ tunnel out of the well even if the applied field is low.

The unit normalised ground state wave functions in the DMQWs with $L_{d}=20 \AA$ corresponding to different electric field strengths are shown in Fig. 7. The wave function without electric field has the peak position at $z=0$ and with higher electric field strength the peak is found to shift in the direction of the electric field. The oscillations in the wave function in the left hand side shows the tunneling of the

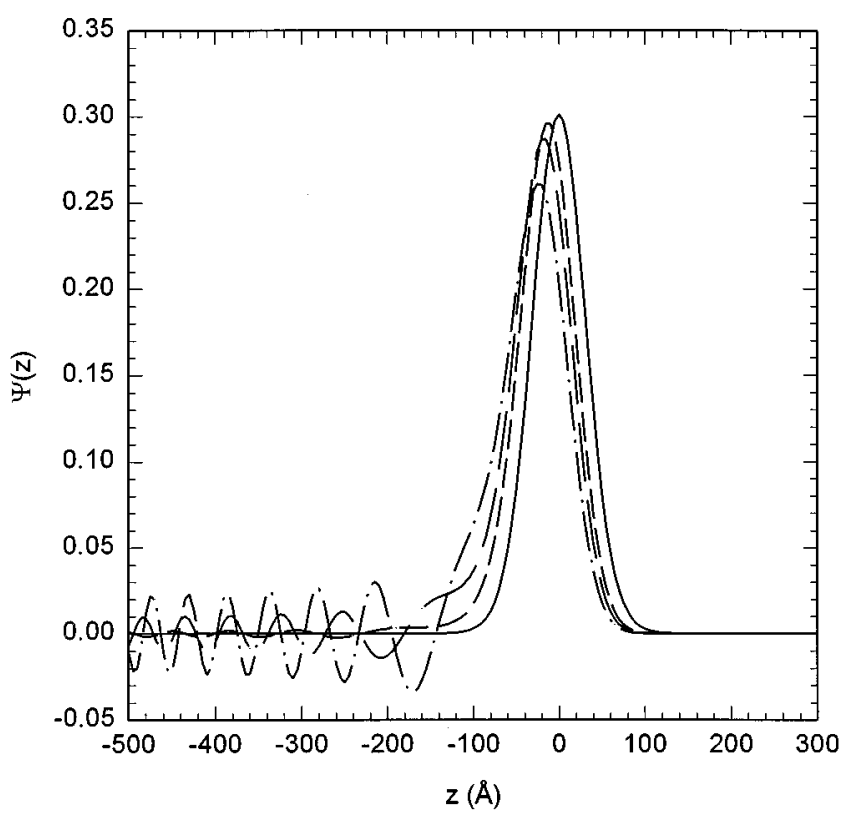

FIG. 7. Wave functions in the DMQW with diffusion constant at $20 \AA$, $x=0.353$ and as-grown well width $100 \AA$ for different applied electric fields are shown here. The solid, short-dashed, long-dashed and dot-dashed lines correspond to wave functions at field strengths $0 \mathrm{kV} / \mathrm{cm}, 150 \mathrm{kV} / \mathrm{cm}, 200$ $\mathrm{kV} / \mathrm{cm}$ and $250 \mathrm{kV} / \mathrm{cm}$ respectively.

wave function out of the well at and above $150 \mathrm{kV} / \mathrm{cm}$. The energy of the electron at $0,150,200$ and $250 \mathrm{kV} / \mathrm{cm}$ are found to be $0.0597,0.0499,0.0415$ and $0.0301 \mathrm{eV}$ respectively. The decrease in the energy level makes the electron quasi-bound to the well for which the amplitude of oscillations increases with the increasing field.

The probability of tunneling for diffusion lengths $20 \AA$ and $40 \AA$ are shown in Figs. 8 and 9 respectively. At $t=0$ the probability of tunneling is initiated because the electric field is applied suddenly at this time. At large $t$ the probability behaves as $1-\exp (-\lambda t)$ with $\lambda$ being the inverse of the mean lifetime. The oscillations in the probability in the initial time are due to the collision of the wave packet with the barrier. The steady slope in $P_{E}(t)$ shows that the reflection of the wave packet is minimised with time. With the applied electric field at $400 \mathrm{kV} / \mathrm{cm}$ the wave packet smoothly tunnels out of the well whereas for $300 \mathrm{kV} / \mathrm{cm}$ and $200 \mathrm{kV} / \mathrm{cm}$ the wave packet shows one collison and five collisions respectively. At the same time the particle oscillates in the well for a long time before escaping out of the well when the applied bias is at $100 \mathrm{kV} / \mathrm{cm}$. But for the well with $L_{d}=40 \AA$ the wave packet tunnels out without any collision for both the applied fields at 150 and $200 \mathrm{kV} / \mathrm{cm}$. At $100 \mathrm{kV} / \mathrm{cm}$ there are only few oscillations. This happens because the barrier height reduces significantly under the application of the field.

The mean lifetimes for the as-grown SQW and DMQWs with different $L_{d}$ are shown in Fig. 10. The lifetimes are obtained by fitting $1-\exp (-\lambda t)$ to the calculated mean lifetimes at large $t$. The mean lifetimes are found to decrease with increasing electric field for all $L_{d}$. The change in the mean lifetimes for the as-grown SQW is slower compared to the DMQW. The electric field reduces the barrier height of 


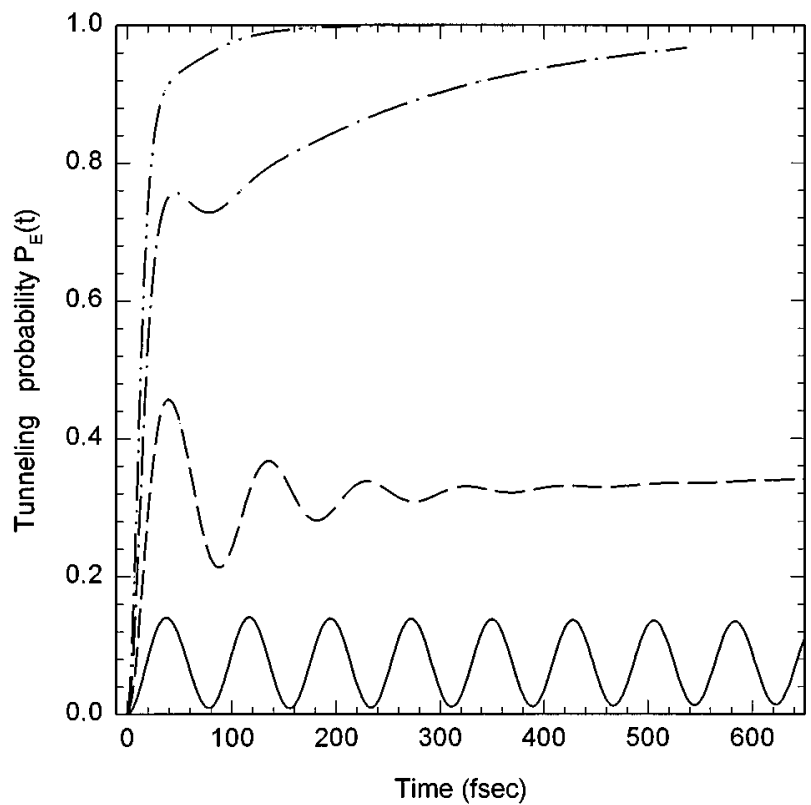

FIG. 8. Probability of tunneling of the particle in the ground state in the DMQW with diffusion length $20 \AA, x=0.353$ and as-grown well width 100 $\AA$ under different applied field strengths. The probability of tunneling at 100 $\mathrm{kV} / \mathrm{cm}, 200 \mathrm{kV} / \mathrm{cm}, 300 \mathrm{kV} / \mathrm{cm}$ and $400 \mathrm{kV} / \mathrm{cm}$ are represented by the solid line, dashed line, dot-dashed and dot-dot-dashed line respectively.

the DMQW more significantly than that of the as-grown SQW. With increasing $L_{d}$ the reduction in the barrier height in the direction of the electric field becomes quite strong so that carriers tunnel out of the well very fast.

The linear, non-linear and total absorption coefficients for the as-grown SQW and the DMQW with $L_{d}$ at $20 \AA$ are

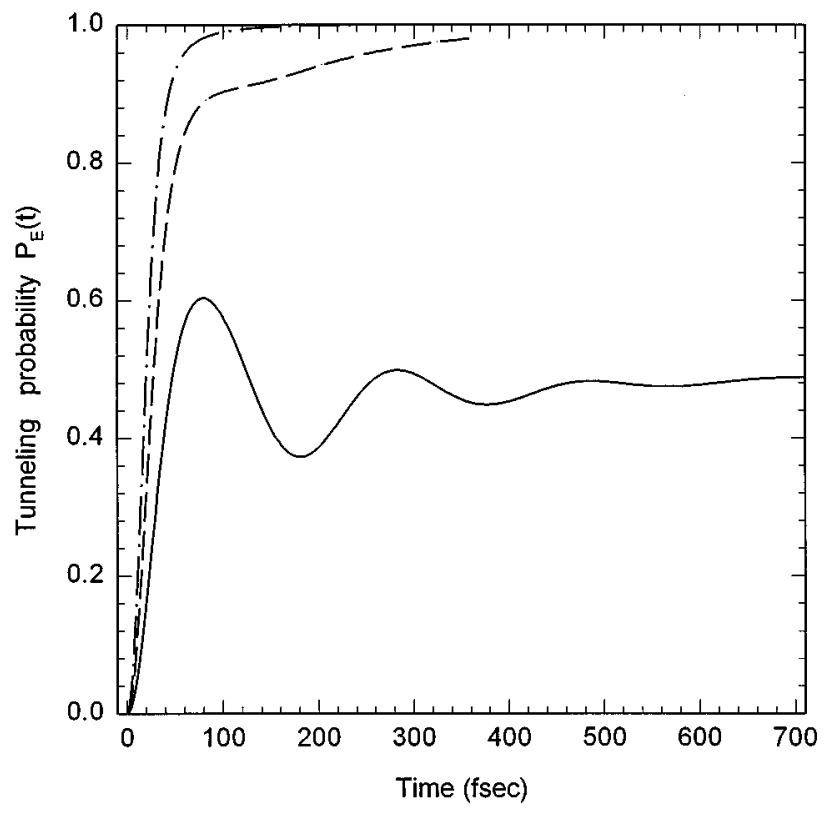

FIG. 9. Probability of tunneling of the particle in the ground state in the DMQW with diffusion length $40 \AA, x=0.353$ and as-grown well width 100 $\AA$ under different applied field field strengths. The probability of tunneling at $100 \mathrm{kV} / \mathrm{cm}, 150 \mathrm{kV} / \mathrm{cm}$ and $200 \mathrm{kV} / \mathrm{cm}$ are represented by the solid line, dashed line, dot-dashed line respectively.

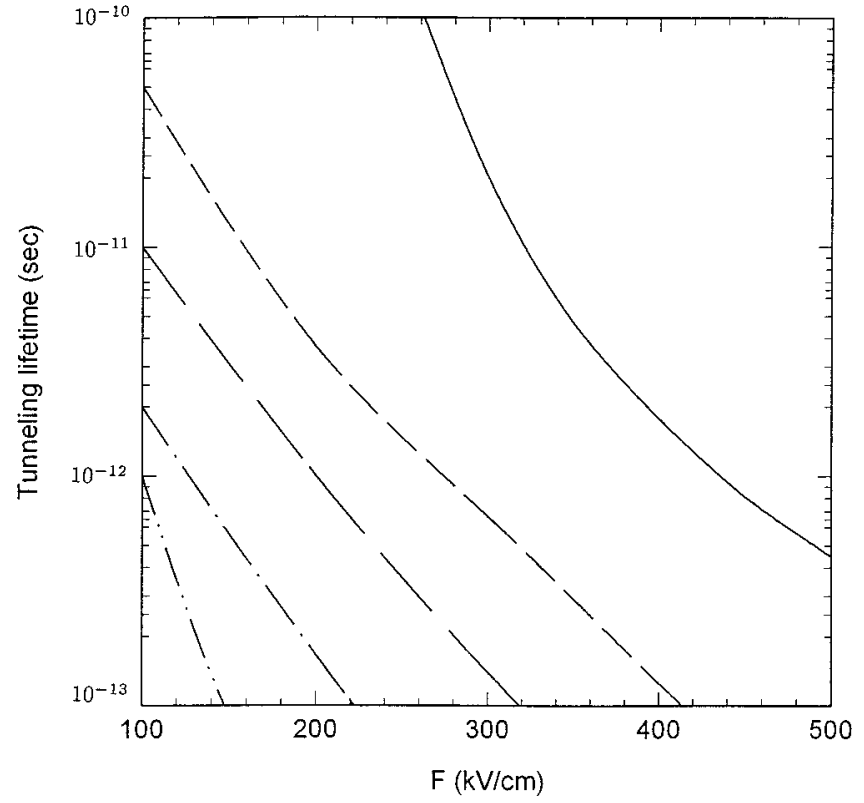

FIG. 10. Mean tunneling lifetimes at different applied fields in the as-grown SQW and DMQW with parameters shown in Fig. 1. The lifetime data at $L_{d}=0 \AA, 10 \AA, 20 \AA, 30 \AA$, and $40 \AA$ are represented by the solid, shortdashed, long-dashed, dot-dashed dot-dot-dashed lines respectively.

plotted in Figs. 11 and 12 respectively. In all cases the absorption is from the initial ground state to the final first excited state. The sample temperature and the incident optical intensity are taken to be $77 \mathrm{~K}$ and $0.5 \mathrm{MW} / \mathrm{cm}^{2}$ respectively. For the as-grown SQW the $E_{i}, E_{f}$ and $E_{F}$ are taken to be $28.3 \mathrm{meV}, 135.23 \mathrm{meV}$ and $23 \mathrm{meV}$ respectively at the field

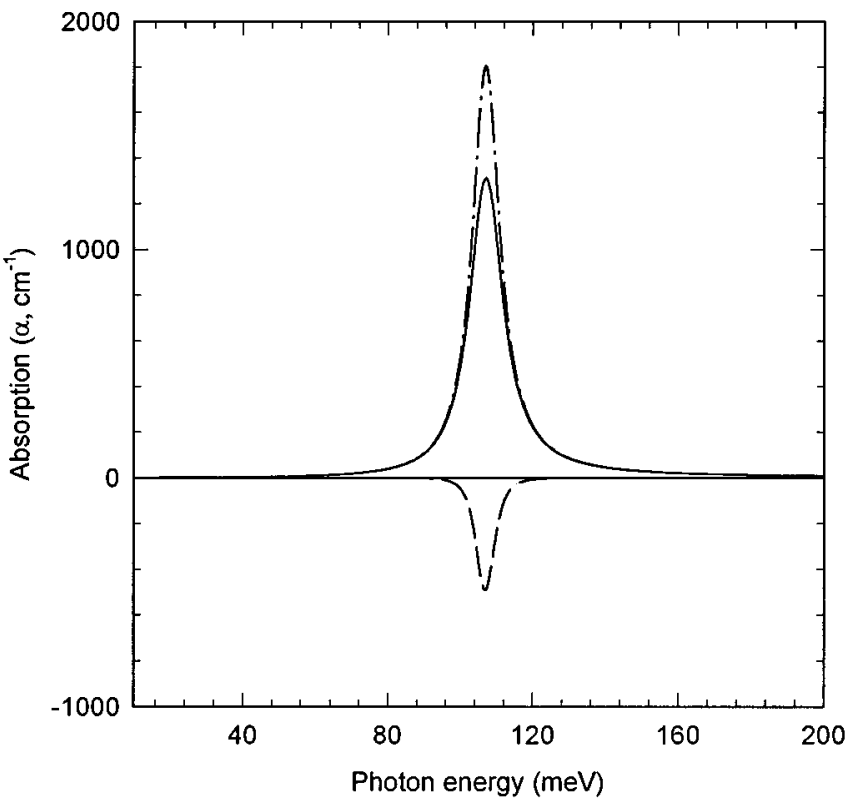

FIG. 11. Linear, nonlinear and total optical absorption coefficients in the as-grown SQW with parameters shown in Fig. 1 under the applied field 100 $\mathrm{kV} / \mathrm{cm}$. The optical intensity is $0.5 \mathrm{MW} / \mathrm{cm}^{2}$ and the sample temperature is $77 \mathrm{~K}$. The linear $\left(\alpha_{1}\right)$, non-linear $\left(\alpha_{3}\right)$ and total $\left(\alpha_{1}+\alpha_{3}\right)$ optical absorption coefficients are represented by dot-dashed, dashed and solid lines respectively. 


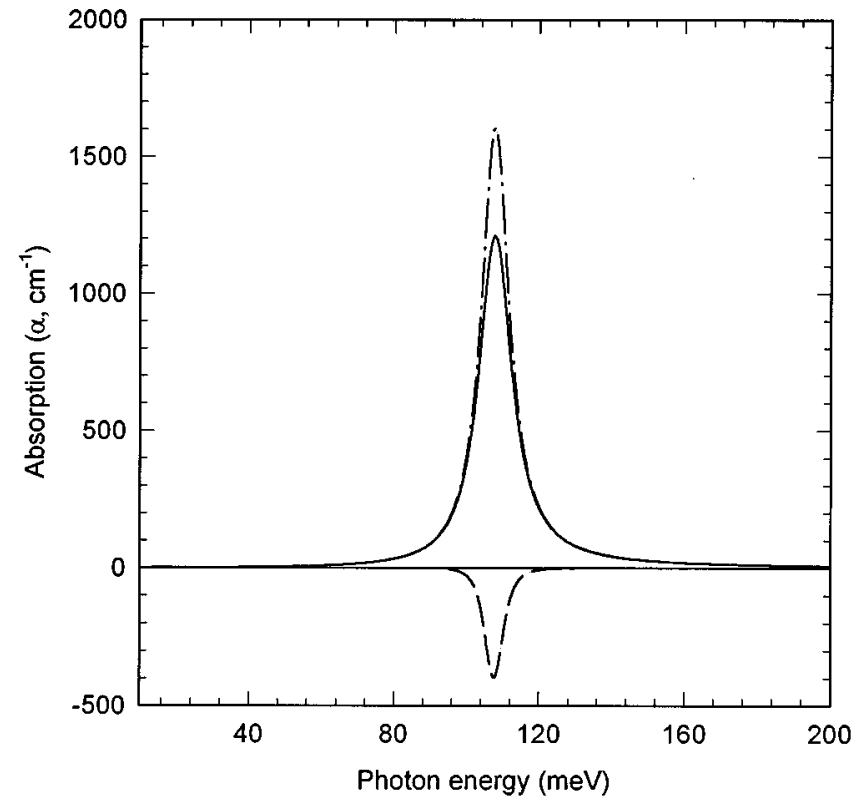

FIG. 12. Linear, nonlinear and total optical absorption coefficients in the DMQW with diffusion length $L_{d}=20 \AA$ and other parameters shown in Fig. 1 under the applied field $100 \mathrm{kV} / \mathrm{cm}$. The intensity of the optical source is $0.5 \mathrm{MW} / \mathrm{cm}^{2}$ and the sample temperature is $77 \mathrm{~K}$. The notations for $\alpha_{1}$, $\alpha_{3}$ and $\alpha_{1}+\alpha_{3}$ are the same as in Fig. 11 .

strength $100 \mathrm{kV} / \mathrm{cm}$. The Fermi energy at $77 \mathrm{~K}$ shows that there are about $3 \times 10^{16} / \mathrm{cm}^{3}$ electrons in the well. The linear $\left[\alpha^{1}(\omega)\right]$ and the non-linear $\left[\alpha^{3}(\omega)\right]$ absorption coefficients are found to be positive and negative in nature respectively. With the present optical intensity the linear part is found to decide the nature of the overall behaviour of the total absorption coefficient. When the optical energy is equal to the difference in energies of the ground and first excited states we obtain the peak in the absorption spectra. For the DMQW with $L_{d}=20 \AA$, the $E_{i}$ and $E_{f}$ are $55.5 \mathrm{meV}$ and $163 \mathrm{meV}$ respectively at $F=100 \mathrm{kV} / \mathrm{cm}$. It is required to solve Poisson equation for a doped QW to get Fermi level. We have taken the Fermi level to be $50 \mathrm{meV}$ which has been chosen by $\mathrm{Li}$, Weiss and Laszcz to study the absorption spectra in the DMQWs at zero electric strength. ${ }^{19}$ The similar strength of the absorption spectra in the DMQW and SQW suggests that the DMQW can be designed to tune a far infrared region.

The changes in the refractive index for the SQW and DMQW with $L_{d}=20 \AA$ are shown in Figs. 13 and 14 respectively. It is clear that the linear and non-linear parts of the refractive index show zero values when the optical energy is equal to the difference in energies of the ground and first excited state. As in the optical absorption the linear and nonlinear parts have opposite sign in the SQW and DMQW at $L_{d}=20 \AA$. The total change in the index of refraction is decided by the linear term only and the non-linear term only decreases the magnitude.

\section{CONCLUSIONS}

In the present work the electron energy levels, wave functions and mean lifetimes in a single DMQW $\mathrm{Al}_{x} \mathrm{Ga}_{1-x} \mathrm{As} / \mathrm{GaAs} / \mathrm{Al}_{x} \mathrm{Ga}_{1-x} \mathrm{As}$ have been studied. The in-

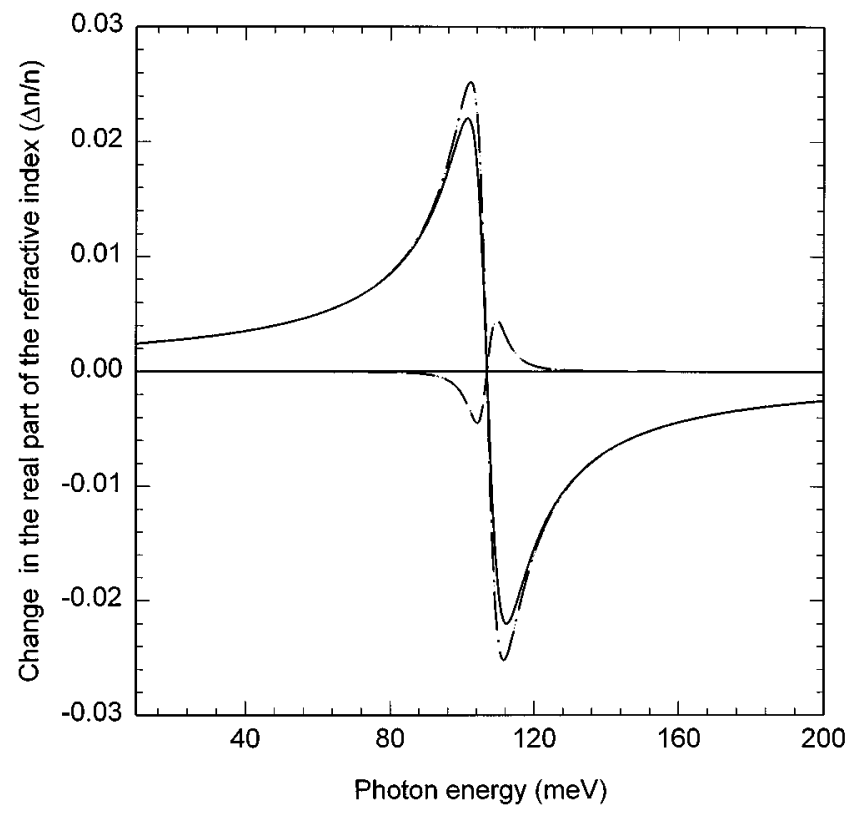

FIG. 13. Linear, nonlinear and total index of refraction in as-grown SQW with parameters shown in Fig. 1 under applied field strength $100 \mathrm{kV} / \mathrm{cm}$. The intensity of the optical source is $0.5 \mathrm{MW} / \mathrm{cm}^{2}$ and the temperature of the sample is $77 \mathrm{~K}$. The notations for the linear, non-linear and total change in the index of refraction are represented by the dot-dashed, dashed and solid lines respectively.

crease in the ground state energy level with the $\mathrm{Al}$ diffusion length up to $20 \AA$ has been explained to arise from the decrease in the well width due to the interdiffusion process. The decrease in energy levels in the DMQW with interdiffusion length more than $20 \AA$ has been attributed to both

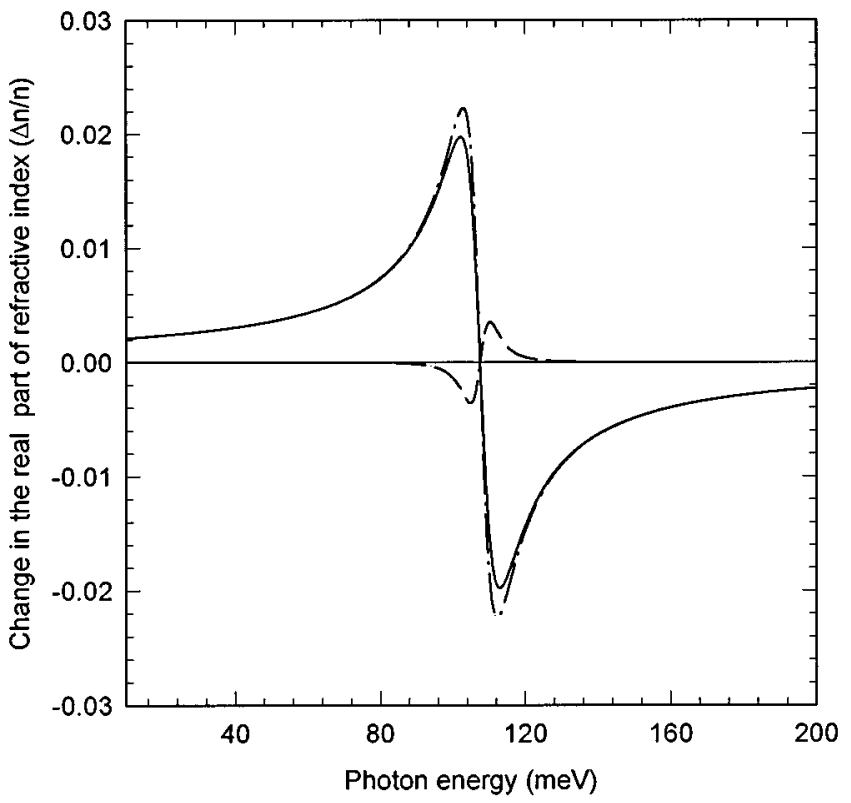

FIG. 14. Linear, nonlinear and total index of refraction in as-grown SQW with parameters shown in Fig. 1 under applied field strength $100 \mathrm{kV} / \mathrm{cm}$. The intensity of the optical source is $0.5 \mathrm{MW} / \mathrm{cm}^{2}$ and the temperature of the sample is $77 \mathrm{~K}$. The notations for the linear, non-linear and total change in the index of refraction are the same as in Fig. 13. 
the increase in the well width and the decrease in the barrier height. The Stark shifts and the probability of tunneling are found to be quite high with wells having $\mathrm{Al}$ diffusion lengths more than $20 \AA$. The optical absorption and the change in refractive index data in the SQW and DMQW show that DMQW can be used as a far IR detector. Our work is done without a full many-body calculation which may be valid when the samples are not doped. In the SQW it has been observed that the doping increases both the intersubband absorption and change in the index of refraction. ${ }^{18}$ In order to get a better picture about the electronic structure of the diffused quantum wells it is necessary to study the many-body problem in these wells. ${ }^{32}$ The photon induced many-body contributions such as depolarisation ${ }^{33}$ and exciton like interactions ${ }^{34}$ between the ground state and the excited states shifts the calculated energy position by a substantial amount. The second and third harmonic generations for a finite parabolic quantum well is theoretically found to be too high. ${ }^{35} \mathrm{It}$ is possible to physically create a parabolic quantum well by suitably controlling the diffusion length. Therefore it is warranted to take up all these calculations which are not available at present.

${ }^{1}$ N. Peyghambarian, S. W. Koch, and A. Mysyrowich, Intoduction to Semiconductor Optics (Prentice Hall, Englewood Cliffs, NJ, 1993).

${ }^{2}$ J. P. Loehr and M. O. Manasreh, Semiconductor Quantum Wells and Superlattices for Long Wavelength Infrared Detectors, edited by M. O. Manasreh (Artech House, 1993).

${ }^{3}$ NATO Advanced Research Workshop in Intersubband Transitions in Quantum Wells, Intersubband Transitions in Quantum Wells (Plenum, New York, 1992).

${ }^{4}$ A. Miller, D. A. B. Miller, and S. D. Smith, Adv. Phys. 30, 697 (1981).

${ }^{5} \mathrm{~W}$. Walukiewicz, Semiconductor Interfaces and Microstructures, edited by C. F. Zhe (World Scientific, Singapore, 1992), p. 1.

${ }^{6}$ L. L. Chang and A. Koma, Appl. Phys. Lett. 29, 138 (1976).

${ }^{7}$ K. Mukai, M. Sugawara, and S. Yamazaki, Phys. Rev. 50, 2273 (1994).
${ }^{8}$ Y. T. Oh, T. W. Kang, C. Y. Hong, K. T. Kim, and T. W. Kim, Solid State Commun. 94, 241 (1995).

${ }^{9}$ T. E. Schlesinger and T. F. Kuech, Appl. Phys. Lett. 49, 519 (1986).

${ }^{10}$ K. Kash, B. Tell, P. Grabbe, E. A. Dobisz, H. G. Graighead, and C. M. Tamargo, J. Appl. Phys. 63, 190 (1988).

${ }^{11}$ D. Ahn and S. L. Chuang, Phys. Rev. B 34, 9034 (1986).

${ }^{12}$ C. Juang, K. J. Kuhn, and R. B. Darling, Phys. Rev. B 41, 12047 (1990).

${ }^{13}$ K. Nakamura, A. Shimizu, M. Koshiba, and K. Hayata, IEEE J. Quantum Electron. 25, 889 (1989).

${ }^{14}$ B. Jonsson and T. Eng, IEEE J. Quantum Electron. 25, 2025 (1990).

${ }^{15}$ A. K. Ghatak, I. C. Goyal, and R. L. Gallawa, IEEE J. Quantum Electron. 26, 305 (1990).

${ }^{16} \mathrm{~S}$. Panda and E. H. Li (unpublished).

${ }^{17}$ D. Ahn and S. L. Chuang, IEEE J. Quantum Electron. QE-23, 2196 (1987).

${ }^{18}$ K. J. Kuhn, G. U. Iyengar, and S. Yee, J. Appl. Phys. 70, 5010 (1991).

${ }^{19}$ E. H. Li, B. L. Weiss, and A. Laszcz, Electron. Lett. 28, 885 (1992).

${ }^{20}$ E. H. Li, B. L. Weiss, and K. S. Chan, Phys. Rev. B 46, 15181 (1992).

${ }^{21}$ I. Lahiri, D. D. Nolte, J. C. P. Chang, J. M. Woodall, and R. M. Melloch, Appl. Phys. Lett. 67, 1244 (1995).

${ }^{22}$ M. Abramowitz and I. A. Stegun, Handbook of Mathematical Functions, Washington DC (National Bureau of Standards, Washington, DC, 1964), p. 446.

${ }^{23}$ D. J. BenDaniel and C. B. Duke, Phys. Rev. B 152, 683 (1966).

${ }^{24}$ See, for example, G. W. Steward, Introduction to Matrix Computations (Academic, New York, 1973), Chap. 7.

${ }^{25}$ J. Kundrotas and A. Dargys, Phys. Status Solidi B 134, 267 (1986).

${ }^{26}$ N. Bloembergen, Nonlinear Optics (Benjamin, New York, 1965), Chap. 2.

${ }^{27}$ L. C. West and R. J. Eglash, Appl. Phys. Lett. 46, 1156 (1983).

${ }^{28}$ W. Walukiewicz, H. E. Ruda, J. Lagowski, and H. C. Gatos, Phys. Rev. B 30, 4571 (1984).

${ }^{29}$ F. Borondo and J. Sánchez-Dehesa, Phys. Rev. B 33, 8758 (1986).

${ }^{30}$ J. A. Proto, J. Sánchez-Dehesa, L. A. Cury, A. Nogare, and J. C. Portal, J. Phys. Condens. Matter 6, 887 (1994).

${ }^{31}$ D. A. V. Miller, D. S. Chemla, T. C. Damen, A. C. Gossard, W. Wiegman, T. H. Wood, and C. A. Burrus, Phys. Rev. B 32, 1043 (1985).

${ }^{32}$ K. M. S. V. Bandara, D. D. Coon, O. Byungsung, Y. F. Lin and M. H. Francombe, Appl. Phys. Lett. 53, 1931 (1988).

${ }^{33}$ S. J. Allen, D. C. Tsui, and B. Vinter, Solid State Commun. 20, 425 (1976).

${ }^{34}$ T. Ando, Z. Phys. B 26, 263 (1977).

${ }^{35}$ Y. Huang and C. Lien, J. Appl. Phys. 75, 3223 (1994). 